\title{
Fragilidade em idosos assistidos por equipes da atenção primária
}

\author{
Frailty among the elderly assisted by primary health care teams
}

Luciana Colares Maia (https://orcid.org/0000-0001-6359-3593) ${ }^{1}$

Edgar Nunes de Moraes (https://orcid.org/0000-0002-8923-1029) ${ }^{2}$

Simone de Melo Costa (http://orcid.org/0000-0002-0266-018X) ${ }^{3}$

Antônio Prates Caldeira (https://orcid.org/0000-0002-9990-9083) ${ }^{4}$
${ }^{1}$ Centro Mais Vida Eny Faria de Oliveira, Hospital Universitário Clemente de Faria, Centro de Ciências Biológicas e da Saúde, Departamento de Clínica Médica, Universidade Estadual de Montes Claros (Unimontes). Av. Dr. Ruy Braga s/n, Vila Mauriceia. 39401-089 Montes Claros MG Brasil. luciana.colares. maia@gmail.com

${ }^{2}$ Núcleo de Geriatria

e Gerontologia,

Departamento de Clínica

Médica, Faculdade de

Medicina, Universidade

Federal de Minas Gerais.

Belo Horizonte MG Brasil.

${ }^{3}$ Centro de Ciências

Biológicas e da Saúde,

Departamento de

Odontologia, Unimontes.

Montes Claros MG Brasil.

${ }^{4}$ Centro de Ciências

Biológicas e da Saúde,

Departamento de Saúde

da Mulher e da Criança,

Unimontes. Montes Claros

MG Brasil.
Abstract The scope of this study was to assess the prevalence and factors associated with frailty among the elderly in Primary Health Care. It is a cross-sectional study carried out with 1750 elderly people in the Southeast of Brazil. The Brazilian Older American Resources and Services Multidimensional Functional Assessment Questionnaire (BOMFAQ) and the Clinical-Functional Vulnerability Index (CFVI) were used for data collection. Descriptive and bivariate analyzes were performed, followed by Poisson regression analysis, with robust variance, to obtain adjusted prevalence ratios (PR). The CFVI identified 357 frail elderly people (20.1\%). The variables that remained statistically associated with frailty after multiple analysis were: single/widowed $(P R=$ 1.05; 95\%CI =1.02-1.07); four years of schooling $(P R=1.05 ; 95 \% C I=1.03-1.08)$; polypathology $(P R=1.05 ; 95 \% C I=1.01-1.09) ;$ polypharmacy $(P R=1.21 ; 95 \% C I=1.17-1.26) ;$ mental disorder $(P R=1.16 ; 95 \% C I=1.12-1.21)$; cognitive impairment ( $P R=1.71$; 95\%CI = 1.57-1.86); Daily Living Activity impairment $(P R=1.12$; $95 \% C I$ $=1.09-1.14)$; falls $(P R=1.06 ; 95 \% C I=1.03$ 1.10); hospitalization $(P R=1.39 ; 95 \% C I=1.27$ 1.52); urinary incontinence and self-perception of health. The conclusion that a significant prevalence of frailty in the study reaffirms the need for a multidimensional approach to the elderly.

Key words Elderly, Frailty, Primary Health Care
Resumo Objetivou-se conhecer a prevalência e os fatores associados à fragilidade em idosos na Atenção Primária à Saúde. Trata-se de estudo transversal realizado com1750 idosos no Sudeste do Brasil. Utilizou-se para coleta o Brazilian Older Americans Resources and Services MultiDimensional Function Assessment Questionnaire (BOMFAQ) e o Índice de Vulnerabilidade Clínico-funcional (IVCF-20). Realizaram-se as análises descritivas e bivariadas, seguidas da análise regressão de Poisson, com variância robusta, para obtenção de razões de prevalência ajustadas. O IVCF-20 identificou 357 idosos frágeis $(20,1 \%)$. As variáveis estatisticamente associadas à fragilidade após análise múltipla foram: estado civil $(R P=1,05 ;$ IC95\% $=1,02-1,07)$, escolaridade $(R P=1,05 ; I C 95 \%=$ 1,03-1,08), polipatologia $(R P=1,05$; IC95\% $=$ $1,01-1,09)$, polifarmácia $(R P=1,21$; IC95\% $=$ $1,17-1,26)_{2}$ transtorno mental $(R P=1,16 ;$ IC95\% $=1,12-1,21)$, comprometimento cognitivo $(R P=$ 1,71; IC95\% = 1,57-1,86), comprometimento de atividades de vida diária $(R P=1,12 ; 1 C 95 \%=$ $1,09-1,14)$, quedas $(R P=1,06 ; I C 95 \%=1,03$ $1,10)$, internação $(R P=1,39 ; I C 95 \%=1,27-1,52)$ e incontinência urinária, autopercepção da saúde. Concluiu-se que a significante prevalência de fragilidade do estudo reitera a necessidade de abordagem multidimensional do idoso.

Palavras-chave Idoso, Fragilidade, Atenção Primária à Saúde 


\section{Introdução}

O envelhecimento populacional é uma realidade mundial que tem grande impacto sobre o perfil epidemiológico e sobre as demandas dos serviços de saúde, constituindo um grande desafio às políticas públicas ${ }^{1}$. O relatório mundial sobre envelhecimento e saúde da Organização Mundial de saúde (OMS) já sinalizava que o número de indivíduos com 60 anos aumentará exponencialmente em $2050^{2}$. Esses dados destacam, por si só, a relevância do tema e a necessidade de estudos que abordam a população idosa.

O envelhecer acarreta mudanças inevitáveis, progressivas, heterogêneas, nem sempre patológicas, mediadas por fatores biopsicossociais que podem, de forma imprevisível, repercutir no processo saúde doença dos idosos ${ }^{2,3}$. Assim, o envelhecimento está profundamente ligado a redução da vitalidade e ao processo de fragilização ${ }^{4,5}$. O acúmulo de condições clínicas e incapacidades podem contribuir para o estado de vulnerabilidade dos idosos, com consequente desenvolvimento da fragilidade e do declínio do estado de saúde $e^{5,6}$.

$\mathrm{Na}$ análise da fragilidade de idosos, alguns autores destacam o fenótipo da fragilidade ${ }^{4}$, enquanto outros ressaltam o modelo de déficits cumulativos, que valoriza todas as condições de saúde (doenças, comorbidades, sinais e sintomas, exames complementares, dentre outros) como fatores que interferem na capacidade intrínseca da pessoa idosa, além das incapacidades funcionais ${ }^{5,7}$. Esta última abordagem utiliza uma definição mais ampla, baseada na Avaliação Geriátrica Compreensiva de rotina, que consiste na contagem de deficiências em várias áreas, incluindo humor, cognição e incontinência 5 . A literatura apresenta ainda outras variações de abordagem, com pesquisadores que consideram os domínios físico, social e psicológico na investigação e na operacionalização da análise de fragilidade em idosos ${ }^{8}$.

De forma consensual, a fragilidade caracteriza-se como um estado clínico dinâmico, multifatorial, que determina o desequilíbrio das reservas homeostáticas associado à redução da capacidade de resposta aos mínimos agravos, desencadeando declínios cumulativos e consequentes situações indesejáveis ao organismo $0^{6,9}$. É considerada uma síndrome geriátrica complexa, que além de demandar uma abordagem multidimensional exige um planejamento criterioso de práticas de cuidados contínuos, integrados e dinâmicos. Ela requer também intervenções capazes de prevenir implicações negativas, como o agravamento das doenças crônicas, perda funcional, quedas e suas consequências, além de institucionalização, hospitalização e morte ${ }^{10-12}$.

A identificação rápida e oportuna de idosos com maior vulnerabilidade ou frágeis pode auxiliar na aplicação de intervenções que são benéficas aos idosos e suas famílias ${ }^{13}$. De forma similar, a identificação dos fatores associados à fragilidade tem o potencial de direcionar políticas públicas de saúde para prevenção de situações críticas com redução dos impactos sobre o sistema de saúde ${ }^{14}$. Esse aspecto é muito importante no contexto da Atenção Primária em Saúde (APS), considerando a necessidade de organizar um modelo de cuidado integrado e centrado nas particularidades das pessoas idosas. As ações específicas desenvolvidas para este usuário exigem o seguimento contínuo desde a entrada do idoso no sistema até a terminalidade da vida, buscando sempre maximizar a qualidade de vida. Assim, é fundamental o reconhecimento precoce dos riscos de fragilização deste segmento etário com intuito de prevenir e identificar os desfechos adversos à saúde pelos profissionais do Sistema Único de Saúde (SUS) $)^{2,15}$.

Existe grande variabilidade nos estudos de prevalência de fragilidade entre idosos, em diversos países, o que define maior necessidade de estudos sobre o tema em contextos diferentes ${ }^{16-18}$. Diversos instrumentos de triagem estão disponíveis na literatura ${ }^{4,18,19}$ para rastrear o idoso vulnerável ou o frágil ${ }^{4,19,20}$. No Brasil, uma ferramenta recentemente elaborada tem sido proposta para avaliação multidimensional e identificação de idosos com maior risco de vulnerabilidade e fragilidade, trata-se do Índice de Vulnerabilidade Clínico-Funcional de 20 itens (IVCF-20) ${ }^{21}$. Esse instrumento tem potencial de auxiliar as equipes da APS na identificação de idosos frágeis para um cuidado mais oportuno. A literatura nacional ainda carece de estudos que se aprofundem na magnitude do problema na rede pública. Assim, este estudo teve como objetivo conhecer a prevalência e os fatores associados à fragilidade em idosos acompanhados por equipes da Atenção Primária à Saúde.

\section{Métodos}

Trata-se de um estudo transversal, realizado com pessoas de 60 anos ou mais, cadastradas e acompanhadas por equipes da Estratégia Saúde da Família (ESF) na região urbana em uma cidade de porte médio do Sudeste brasileiro. A cidade 
conta com uma população de aproximadamente 400 mil habitantes e tem mais de $80 \%$ de cobertura assistencial pelas equipes da ESF. O município é o principal polo urbano da região, tem um Índice de Desenvolvimento Humano Municipal (IDH-M) igual a 0,770 e, em 2013 registrava uma expectativa de vida ao nascer de 77,1 anos.

O cálculo amostral para participação da investigação deu-se a partir da fórmula para população infinita. Para a definição do tamanho da amostra, considerou-se, para o local do estudo, uma população estimada 8,2\% de idosos, correspondendo a 33.930 pessoas de 60 anos ou mais. A frequência do evento estudado foi considerada $50 \%$, por se tratar de uma frequência conservadora e que produz um maior número amostral. Estimou-se um nível de confiança 95\% e uma margem de erro amostral de 3\%. O processo de amostragem foi por conglomerados em dois estágios. No primeiro estágio foram sorteados quatro entre os 12 polos regionais de saúde do município e, em cada polo realizou-se o sorteio das equipes da ESF e respectivas áreas de abrangência. Para cada território, sortearam-se as microáreas de saúde, onde todos os idosos foram considerados elegíveis para o estudo. O tamanho amostral calculado mínimo foi 1.035 indivíduos, porém, considerando-se o processo de amostragem por conglomerados, esse número foi multiplicado por um fator de correção para o efeito do desenho igual a 1,5 e acrescido de $10 \%$ para eventuais perdas. Assim, o número mínimo de participantes do estudo foi calculado em 1.708 indivíduos.

A coleta dos dados aconteceu no domicílio, as entrevistas foram realizadas no período matutino, vespertino ou noturno e em todos os dias da semana. A equipe de entrevistadores era formada por estudantes do curso de medicina e enfermeiras e foi devidamente treinada. Previamente ao início da pesquisa, foi conduzido um projeto-piloto em área distinta daquelas selecionadas para a pesquisa, para calibração final dos entrevistadores e os dados não foram incluídos no trabalho final.

Aqueles idosos incapacitados de responder aos questionários e que não tinham um cuidador/responsável disponível durante as visitas foram excluídos do estudo. Foram consideradas perdas aqueles indivíduos que não estavam no domicílio para colaborarem com a investigação em pelo menos três visitas em dias e horários diferentes, mesmo após o agendamento prévio.

As informações da pesquisa foram obtidas pela aplicação de dois questionários: o Brazilian
Older Americans Resources and Services MultiDimensional Function Assesment Questionnaire (BOMFAQ) que é uma versão brasileira do $\mathrm{Ol}$ der Americans Resources and Services (OARS) e o IVCF-20 (Índice de Vulnerabilidade Clínicofuncional). O BOMFAQ é uma avaliação multidimensional do idoso adaptada e validada no Brasil. É um instrumento constituído de questões que avaliam: dados sociodemográficos, capacidade funcional utilizando as atividades de vida diária (AVD), saúde física, saúde mental através do Mini Mental State Examination (MMSE) e do Questionário de Rastreamento Psicogeriátrico (QRP), além da integração social ${ }^{22}$.

O IVCF-20 é um questionário para triagem inicial de idosos potencialmente frágeis, de caráter multidimensional, simples e de rápida aplicação (5 a 10 minutos). Desenvolvido e validado no Brasil, ele é capaz de identificar o indivíduo em risco de declínio clínico-funcional. O instrumento engloba oito dimensões consideradas preditoras de perda clínico-funcional e óbito em pessoas idosas: a idade, a autopercepção da saúde, as atividades de vida diária (três AVD instrumentais e uma AVD básica), a cognição, o humor/comportamento, a mobilidade (alcance, preensão e pinça; capacidade aeróbica/muscular; marcha e continência esfincteriana), a comunicação (visão e audição) e a presença de comorbidades múltiplas, representada por polipatologia, polifarmácia e/ou internação recente. Apresenta pontuação de 0 a 40, sendo que os idosos com escore final de 0 a 6 têm alta probabilidade de serem robustos, enquanto aqueles com a pontuação de 7 a 14 préfrágeis, e o escore de 15 ou mais pontos, tem uma elevada probabilidade de fragilidade ${ }^{21}$.

$\mathrm{O}$ atual estudo contemplou as seguintes variáveis: sexo, estado civil (solteiro/viúvo/divorciado e casado/união estável), escolaridade (zero a quatro anos e cinco ou mais), autopercepção de saúde (positiva e negativa), polifármacia (sim e não), polipatologias (sim e não), possibilidade de transtorno mental pelo QRP (sim e não), provável comprometimento cognitivo pelo MEEM ( $\operatorname{sim}$ e não), AVDs comprometidas ( $\operatorname{sim}$ e não), histórico de quedas no último ano ( $\operatorname{sim}$ e não), internação nos últimos seis meses (sim e não) e incontinência urinária ( $\operatorname{sim}$ e não). O IVCF20 foi considerado como variável dependente, sendo categorizada em $\geq 15$ (idoso frágil) e $<15$ (idoso não frágil).

Para análise dos dados, inicialmente foi realizada a estatística descritiva, seguida de análise bivariada. Todas as variáveis independentes associadas com maior risco de fragilidade (escore do 
IVCF- $20 \geq 15)$ até o nível de $20 \%(\mathrm{p} \leq 0,20)$ foram submetidas à análise múltipla pela Regressão de Poisson, com variância robusta, para a obtenção das razões de prevalências (RP) e intervalos de confiança (IC95\%) ajustados. O método adotado para a entrada de variáveis na análise foi Stepwise, ou seja, todas as variáveis independentes foram inseridas no modelo, com retirada de uma a uma até permanecer no modelo final apenas as variáveis associadas ao nível de $5 \%(\mathrm{p}<0,05)$, Backward deletion. Considerando o delineamento amostral por conglomerado que foi realizado, todas as análises foram realizadas empregando-se fatores naturais de expansão, que são pesos diferenciados para os elementos da amostra, para compensar probabilidades desiguais de seleção. Os dados foram processados por meio do software IBM ${ }^{\circledR}$ SPSS $^{\circledR}$, versão 22.0 .

Cada participante foi devidamente informado sobre a pesquisa e apresentou sua aquiescência, através da assinatura do Termo de Consentimento Livre e Esclarecido (TCLE). Para os que não sabiam assinar, foi realizada a coleta de digitais em campo específico do TCLE. Foi garantido também o anonimato e a confidencialidade de todas as informações fornecidas, que foram utilizadas exclusivamente para fins científicos. $\mathrm{O}$ projeto de pesquisa foi aprovado por Comitê de Ética em Pesquisa devidamente credenciado pela Comissão Nacional de Ética em Pesquisa.

\section{Resultados}

Foram entrevistadas 1.750 idosos, com idade entre 60 e 107 anos, sendo a média igual a 71,6 anos (DP: $\pm 8,6)$. Foram registradas 36 perdas/recusas ao longo da coleta de dados. Entre os participantes a maioria era do sexo feminino $(63,4 \%)$, com escolaridade entre um a quatro anos $(56,8 \%)$. As principais características demográficas e socioeconômicas do grupo obtidas a partir do BOMFAQ estão disponíveis na Tabela 1.

A análise da fragilidade pelo IVCF-20 revelou que $48,7 \%$ dos idosos foram considerados robustos, $32,2 \%$ eram os pré-frágeis, e $20,1 \%$ foram avaliados como os frágeis. Em relação às condições de saúde dentre as variáveis independentes, verificou-se que $28,8 \%$ dos idosos apresentavam uma percepção negativa acerca da própria saúde, avaliando-a como ruim ou péssima; $32,2 \%$ faziam uso de cinco ou mais medicamentos e $27,6 \%$ referiam ter mais de cinco doenças.

Os dados coletados possibilitaram também o reconhecimento de transtornos mentais e de
Tabela 1. Caracterização demográfica e socioeconômica de idosos assistidos por equipes da Estratégia Saúde da Família. Montes Claros-MG, Brasil, 2017.

\begin{tabular}{|c|c|c|c|}
\hline Variáveis & $\mathbf{N}$ & $\%$ & $\%^{*}$ \\
\hline \multicolumn{4}{|l|}{ Sexo } \\
\hline Feminino & 1109 & 63,4 & 63,4 \\
\hline Masculino & 641 & 36,6 & 36,6 \\
\hline \multicolumn{4}{|l|}{ Faixa etária } \\
\hline$\geq 85$ anos & 172 & 9,8 & 9,6 \\
\hline 75 a 84 anos & 396 & 22,6 & 22,5 \\
\hline 60 a 74 anos & 1182 & 67,6 & 67,9 \\
\hline \multicolumn{4}{|l|}{ Estado civil } \\
\hline Solteiro & 159 & 9,1 & 9,1 \\
\hline Casado/união estável & 946 & 54,0 & 54,2 \\
\hline Viúvo & 493 & 28,2 & 28,0 \\
\hline Divorciado & 152 & 8,7 & 8,7 \\
\hline \multicolumn{4}{|l|}{ Escolaridade } \\
\hline Analfabeto & 201 & 11,5 & 11,1 \\
\hline 1 a 4 anos & 995 & 56,8 & 56,8 \\
\hline 5 a 8 anos & 230 & 13,2 & 13,4 \\
\hline$>8$ anos & 324 & 18,5 & 18,7 \\
\hline \multicolumn{4}{|c|}{$\begin{array}{l}\text { Renda familiar (salário mínimo } \\
=\mathrm{R} \$ 937,00 \text { ) }\end{array}$} \\
\hline$>2$ salários mínimos & 598 & 34,2 & 34,4 \\
\hline$\leq 2$ salários mínimos & 1053 & 60,1 & 65,6 \\
\hline Sem informações & 99 & 5,7 & \\
\hline \multicolumn{4}{|l|}{ Arranjo domiciliar } \\
\hline Vive sozinho & 175 & 10,0 & 9,9 \\
\hline Vive acompanhado & 1575 & 90,0 & 90,1 \\
\hline
\end{tabular}

comprometimento cognitivo com prevalências, respectivas de $25,5 \%$ e $11,4 \%$. O prejuízo de alguma atividade de vida diária (AVD) foi detectado em 55,9\% dos indivíduos, sendo que 31,0\% dos idosos investigados tinham quatro ou mais AVDs comprometidas (Tabela 2).

A Tabela 3 apresenta os resultados das análises bivariadas e múltipla. As variáveis que permaneceram estatisticamente associadas à fragilidade após análise múltipla foram: estado civil, escolaridade, autopercepção de saúde, polifarmácia, polipatologia, transtorno mental, comprometimento cognitivo, comprometimento das atividades de vida diária, registro de quedas nos últimos 12 meses, registro de internação nos últimos seis meses e incontinência urinária. 
Tabela 2. Caracterização de aspectos relacionados à saúde de idosos assistidos por equipes da Estratégia Saúde da Família. Montes Claros-MG, Brasil, 2017.

\begin{tabular}{|c|c|c|c|}
\hline Variáveis & $\mathbf{n}$ & $\%$ & $\%^{*}$ \\
\hline \multicolumn{4}{|l|}{ IVCF- $20^{* *}$} \\
\hline Robustos & 845 & 48,3 & 48,7 \\
\hline Pré-frágil & 566 & 31,3 & 31,2 \\
\hline Frágil & 357 & 20,4 & 20,1 \\
\hline \multicolumn{4}{|l|}{ Autopercepção de saúde } \\
\hline Ótima & 211 & 12,1 & 12,3 \\
\hline Boa & 1028 & 58,7 & 58,9 \\
\hline Ruim & 376 & 21,5 & 21,2 \\
\hline Péssima & 135 & 7,7 & 7,6 \\
\hline \multicolumn{4}{|c|}{ Número de medicamentos em uso } \\
\hline$\geq 5$ & 566 & 32,4 & 32,2 \\
\hline $1-4$ & 939 & 53,6 & 53,5 \\
\hline Nenhum & 245 & 14,0 & 14,3 \\
\hline \multicolumn{4}{|c|}{ Polipatologia (cinco ou mais doenças) } \\
\hline Sim & 486 & 27,8 & 27,6 \\
\hline Não & 1264 & 72,2 & 72,4 \\
\hline \multicolumn{4}{|c|}{ Transtorno mental (QRP) $)^{\star * *}$} \\
\hline Sim & 455 & 26,0 & 25,5 \\
\hline Não & 1263 & 72,7 & 73,2 \\
\hline Sem informações & 23 & 1,3 & 1,3 \\
\hline \multicolumn{4}{|c|}{ Provável comprometimento cognitivo (MEEM) $)^{\star * \star *}$} \\
\hline Sim & 201 & 11,5 & 11,4 \\
\hline Não & 1545 & 88,3 & 88,3 \\
\hline Sem medida & 4 & 0,2 & 0,2 \\
\hline \multicolumn{4}{|c|}{ Atividades de vida diária comprometidas } \\
\hline$\geq 7$ & 315 & 18,0 & 18,5 \\
\hline $4-6$ & 217 & 12,4 & 12,5 \\
\hline $1-3$ & 460 & 26,3 & 24,8 \\
\hline Nenhuma & 757 & 43,3 & 44,1 \\
\hline \multicolumn{4}{|l|}{ Queda no último ano } \\
\hline Sim & 534 & 30,5 & 30,6 \\
\hline Não & 1216 & 69,5 & 69,4 \\
\hline \multicolumn{4}{|c|}{ Internação nos últimos seis meses } \\
\hline Sim & 121 & 6,9 & 6,9 \\
\hline Não & 1639 & 93,1 & 93,1 \\
\hline \multicolumn{4}{|c|}{ Condições clínicas autorrelatadas } \\
\hline $\begin{array}{l}\text { Hipertensão arterial } \\
\text { sistêmica }\end{array}$ & 1238 & 70,7 & 70,3 \\
\hline Diabetes & 362 & 20,7 & 20,7 \\
\hline Incontinência urinária & 299 & 17,1 & 16,9 \\
\hline
\end{tabular}

\section{Discussão}

No presente estudo verificou-se que um quinto dos idosos avaliados apresentava algum grau de fragilidade. A literatura registra valores muito diversos em estudos similares, com ampla variação de $4 \%$ a $42,6 \% \%^{7,9,17,23-25}$. É importante destacar que discrepantes resultados dependem da população avaliada e da metodologia utilizada nos trabalhos, e traduzem também, a inexistência de instrumento internacionalmente padronizado para mensuração dessa condição. A diversidade de instrumentos para o rastreamento da fragilidade, suas particularidades e dimensões abordadas justificam, por si, os resultados distintos.

A identificação precoce de fragilização e o acompanhamento constante dos usuários na rede integrada permite a antecipação dos agravos, a reabilitação precoce e reduz o impacto das doenças crônicas na funcionalidade da pessoa idosa ${ }^{1,7}$. O IVCF-20, utilizado neste estudo, foi recentemente desenvolvido e validado no Brasil, e apresentou boa correlação com a Avaliação Geriátrica Ampla (AGA) e também um alto grau de validade e confiabilidade ${ }^{21}$. Esta ferramenta simples, de caráter multidimensional e de fácil aplicabilidade pode ser usada por profissionais não especialistas na estratificação do idoso frágil.

As variáveis deste trabalho relacionadas à maior frequência de fragilidade foram o estado civil solteiro ou viúvo, a baixa escolaridade, a presença de polipatologias e de polifarmácia, o transtorno mental, o comprometimento cognitivo e de atividades de vida diária (AVD), o registro de quedas nos últimos 12 meses, o relato de internação nos últimos seis meses e a incontinência urinária, além da autopercepção negativa da saúde.

$\mathrm{Na}$ análise dos fatores associados à maior fragilidade entre os idosos, destaca-se que não ter cônjuge ou companheiro representa um fator de risco. Este aspecto também é mostrado em outros trabalhos ${ }^{24-26}$. Em pesquisa conduzida no Rio de janeiro registrou-se que os idosos casados parecem ter um envelhecimento bem sucedido ${ }^{27}$.

A escolaridade e a polipatologia também expressaram associação significativa com fragilidade no modelo final da presente investigação, como observado em outros estudos ${ }^{23-25,28}$. A presença de diversas comorbidades e baixa escolaridade corroboram negativamente com a evolução da vulnerabilidade no individuo ${ }^{24,25}$. Igualmente o idoso frágil tem maior possibilidade de apresentar múltiplas doenças, maior grau de dependência funcional e polifarmácia ${ }^{26}$. 
Tabela 3. Análise bivariada e múltipla (Regressão de Poisson) para variáveis associadas à fragilidade, segundo IVCF-20* para idosos assistidos por equipes da Estratégia Saúde da Família. Montes Claros-MG, Brasil, 2017.

\begin{tabular}{|c|c|c|c|c|c|c|c|c|}
\hline \multirow[t]{2}{*}{ Variáveis } & \multicolumn{2}{|c|}{$\begin{array}{l}{ }^{*} \text { IVCF- } \\
20 \geq 15\end{array}$} & \multicolumn{2}{|c|}{$\begin{array}{l}\text { IVCF- } \\
20<15\end{array}$} & \multicolumn{2}{|c|}{ Análise bivariada } & \multicolumn{2}{|c|}{ Análise múltipla } \\
\hline & $\mathbf{n}$ & $\%$ & $\mathbf{n}$ & $\%$ & p & $\operatorname{RP}(\mathrm{IC} 95 \%)^{\star *}$ & $\mathbf{p}$ & RP(IC95\%) \\
\hline Sexo & & & & & $<0,0001$ & & 0,5460 & \\
\hline Masculino & 102 & 28,1 & 539 & 38,7 & & 1,0 & & 1,0 \\
\hline Feminino & 255 & 71,9 & 854 & 61,3 & & $1,47(1,31-1,69)$ & & $0,99(0,97-1,02)$ \\
\hline Estado Civil & & & & & $<0,0001$ & & $<0,0001$ & \\
\hline Casado/União estável & 143 & 40,1 & 801 & 57,7 & & 1,0 & & 1,0 \\
\hline Solteiro/Viúvo/Divorciado & 214 & 59,9 & 590 & 42,3 & & $1,77(1,58-1,97)$ & & $1,05(1,02-1,07)$ \\
\hline Escolaridade & & & & & $<0,0001$ & & $<0,0001$ & \\
\hline$\geq 5$ anos & 74 & 20,8 & 480 & 35,0 & & 1,0 & & 1,0 \\
\hline 0 a 4 anos & 283 & 79,2 & 911 & 65,0 & & $1,80(1,57-2,06)$ & & $1,08(1,03-1,14)$ \\
\hline Polipatologia & & & & & $<0,0001$ & & $<0,0110$ & \\
\hline Não & 166 & 46,4 & 1097 & 79,0 & & 1,0 & & 1,0 \\
\hline Sim & 191 & 54,6 & 295 & 21,0 & & $3,04(2,73-3,37)$ & & $1,05(1,01-1,09)$ \\
\hline Polifarmácia & & & & & $<0,0001$ & & $<0,0001$ & \\
\hline Não & 138 & 39,0 & 1048 & 75,6 & & 1,0 & & 1,0 \\
\hline Sim & 216 & 61,0 & 340 & 24,4 & & $3,36(3,01-3,75)$ & & $1,21(1,17-1,26)$ \\
\hline Transtorno mental $(\mathrm{QRP})^{* * *}$ & & & & & $<0,0001$ & & $<0,0001$ & \\
\hline Não & 163 & 49,1 & 1108 & 80,1 & & 1,0 & & 1,0 \\
\hline Sim & 173 & 50,9 & 282 & 19,9 & & $2,97(2,67-3,31)$ & & $1,16(1,12-1,21)$ \\
\hline $\begin{array}{l}\text { Comprometimento Cognitivo } \\
(\mathrm{MEEM}) \dagger\end{array}$ & & & & & $<0,0001$ & & $<0,0001$ & \\
\hline Não & 234 & 65,7 & 1311 & 94,2 & & 1,0 & & 1,0 \\
\hline Sim & 122 & 34,3 & 79 & 5,8 & & $4,00(3,64-4,40)$ & & $1,71(1,57-1,86)$ \\
\hline AVD $\dagger \dagger$ comprometidas & & & & & $<0,0001$ & & $<0,0001$ & \\
\hline Não & 28 & 7,6 & 729 & 52,2 & & 1,0 & & 1,0 \\
\hline Sim & 329 & 92,4 & 663 & 47,8 & & $9,18(7,36-11,43)$ & & $1,12(1,09-1,14)$ \\
\hline Quedas no último ano & & & & & $<0,0001$ & & $<0,0001$ & \\
\hline Não & 195 & 54,5 & 1021 & 73,2 & & 1,0 & & 1,0 \\
\hline Sim & 162 & 45,5 & 371 & 26,8 & & $1,90(1,71-2,11)$ & & $1,06(1,03-1,10)$ \\
\hline $\begin{array}{l}\text { Internação nos últimos seis } \\
\text { meses }\end{array}$ & & & & & $<0,0001$ & & $<0,0001$ & \\
\hline Não & 296 & 82,7 & 1332 & 95,7 & & 1,0 & & 1,0 \\
\hline Sim & 61 & 17,3 & 60 & 4,3 & & $2,80(2,49-3,16)$ & & $1,39(1,27-1,52)$ \\
\hline \multicolumn{9}{|l|}{ Condições autorrelatadas } \\
\hline Hipertensão Arterial & & & & & $<0,0001$ & & $<0,6730$ & \\
\hline Positiva & 163 & 46,0 & 1075 & 77,5 & & 1,0 & & 1,0 \\
\hline Negativa & 194 & 54,0 & 317 & 22,5 & & $2,89(2,60-3,21)$ & & $1,13(1,09-1,17)$ \\
\hline Diabetes Mellitus & & & & & $<0,0001$ & & $<0,9850$ & \\
\hline Não & 75 & 20,6 & 434 & 29,5 & & 1,0 & & 1,0 \\
\hline Sim & 281 & 79,4 & 957 & 70,5 & & $1,61(1,41-1,85)$ & & $1,00(0,94-1,02)$ \\
\hline Incontinência Urinária & & & & & $<0,0001$ & & $<0,0001$ & \\
\hline Não & 211 & 59,3 & 1237 & 89,0 & & 1,0 & & 1,0 \\
\hline Sim & 145 & 40,7 & 154 & 11,0 & & $3,33(2,81-3,95)$ & & $1,30(1,23-1,38)$ \\
\hline Autopercepção da saúde & & & & & $<0,0001$ & & $<0,0001$ & \\
\hline Positiva & 163 & 46,0 & 1075 & 77,5 & & 1,0 & & 1,0 \\
\hline Negativa & 194 & 54,0 & 317 & 22,5 & & $2,89(2,60-3,21)$ & & $1,13(1,09-1,17)$ \\
\hline
\end{tabular}


Em relação à polifarmácia, consumo regular de cinco ou mais medicamentos, trata-se de uma questão primordial e comum na população idosa, ocorrendo muitas vezes associada à presença de múltiplas doenças crônicas ${ }^{28,29}$. Outros autores também têm registrado a polifarmácia associada à síndrome de fragilidade ${ }^{29-32}$. Observa-se que os estudos relacionados ao uso de medicamentos e seus riscos em idosos na atenção primária ainda são deficientes ${ }^{30-34}$. A polifarmácia e a fragilidade são condições interligadas e complexas, potencialmente modificáveis que merecem destaque especialmente devido ao risco do uso inapropriado e abusivo de medicamentos. No organismo do idoso, a interação entre diversos medicamentos pode ser mais danosa do que no jovem, determinando maior vulnerabilidade e riscos de desfechos negativos para os usuários ${ }^{28,31-33}$. Ademais, a polifarmácia e a iatrogenia podem apresentar relação direta com a polipatologia, bem com o aumento do risco de fragilidade e perda funcional na pessoa idosa ${ }^{29}$.

Os distúrbios mentais são condições clínicas comuns, principalmente com o avançar da idade, e expõem o idoso a estado de maior vulnerabilidade, com consequente prejuízo da saúde e qualidade de vida ${ }^{2,26,27,33}$. Por outro lado, a fragilidade também implica em desenvolvimento e perpetuação de sintomas depressivos ${ }^{26,35}$. Alguns autores já apontaram esta relação crescente e discrepante de transtornos mentais com fragilidade, especialmente em relação à sintomatologia depressi$\mathrm{va}^{23,35,36}$. Este panorama exige atenção na avaliação da saúde mental dos idosos, com intuito de favorecer o desenvolvimento de ações e integração específica nos diferentes níveis do sistema de saúde.

O comprometimento cognitivo, outra condição correlacionada à saúde mental nos idosos, também se mostrou associado à fragilidade entre os participantes da pesquisa, o que está em consonância com a literatura ${ }^{28,37}$. No entanto, existem limitações em discutir este assunto devido à diversidade das versões do instrumento utilizado nas pesquisas. Existe grande variabilidade na literatura nacional, por exemplo, em relação às particularidades metodológicas no processo de avaliação do desempenho cognitivo de idosos ${ }^{38}$. Ainda assim, as queixas cognitivas não podem ser ignoradas e devem ser rastreadas, dado que o desempenho cognitivo e a fragilidade apresentam-se fortemente inter-relacionados, merecendo especial atenção na abordagem da funcionalidade dos idosos ${ }^{39}$.

Outro aspecto muito relevante na saúde e na qualidade de vida do idoso é a capacidade fun- cional. Neste estudo a independência funcional foi analisada por meio do levantamento do número das atividades de vida diária comprometidas e registrou-se associação importante entre o comprometimento funcional e maior risco de fragilidade, o que está em acordo com outros estudos $^{5-7,13,17,23,25,33,40}$. A síndrome da fragilidade provoca vulnerabilidade física e psicológica, podendo gerar o prejuízo das atividades diárias com aumento do risco de incapacidade funcional gerando mais fragilidade e vice-versa ${ }^{5-7,13,24,40}$.

Neste trabalho, o registro de quedas no último ano também se mostrou associado à fragilidade no modelo ajustado. Na literatura verificase que a história de quedas é também frequentemente associada com a fragilidade e incapacidades $^{11,12,23,25}$. Outra variável que apresentou associação estatística significante com a fragilidade após a análise de regressão foi o registro de internação hospitalar nos últimos seis meses. Essas duas condições são consideradas na literatura ora como causa, ora como consequência do quadro de fragilidade ${ }^{11,12}$. Efetivamente, é razoável admitir que o idoso frágil seja mais vulnerável a episódios de quedas e de internações hospitalares $^{2,25,33}$. De forma similar, as consequências de quedas mais severas podem implicar em fraturas, cirurgias ou internações hospitalares que fragilizam o idoso, com impacto negativo ${ }^{5,11,12,28}$.

Dentre as condições clínicas autorrelatadas, apenas a incontinência urinária (IU) manteve-se também associada à fragilidade no modelo final. Ela é considerada uma importante síndrome geriátrica, além de ser uma circunstância clínica estigmatizante, muitas vezes negligenciada, e capaz de desencadear comprometimento funcional nos $\operatorname{idosos}^{28,41}$. Há escassez de estudos correlacionando IU e fragilidade na literatura, o que denota a necessidade de maior investigação sobre as repercussões negativas deste problema na saúde dos idosos, além da possibilidade de prevenção e tratamento ${ }^{41}$.

A autopercepção da saúde é um indicador de qualidade de vida usado em vários estudos epidemiológicos, que também se mostrou associada à fragilidade para a população avaliada, o que é consonante com outros estudos ${ }^{24,28,42,43}$. Portanto, o julgamento negativo da saúde relaciona-se bidirecionalmente com os sucessivos eventos desfavoráveis e a debilidade progressiva dos idosos somados ao alto risco de vulnerabilidade entre os indivíduos frágeis.

Existem divergências na literatura sobre a relação entre fragilidade e sexo. Este trabalho, em consonância com alguns estudos ${ }^{17,36,40}$, não 
apresentou associação significativa com a fragilidade e sexo feminino, em contradição com outras investigações ${ }^{24,44}$. Não existe uma explicação clara para o fato, mas é possível que as diferenças registradas pelos estudos, retratem que as mulheres, apesar de destacadas com maior risco de fragilização, apresentam no curso dinâmico da fragilidade, as taxas mais rápidas de mudanças em comparação com o sexo masculino e menor mortalidade? .

Em síntese, é importante destacar que a síndrome da fragilidade é um estado multidimensional que resulta da combinação de diversos fatores de risco subjacentes ou coincidentes ${ }^{5,6,9,33}$. Desta forma, é comum que exista sobreposição e até mesmo superestimação da força dessa relação, que além de frequente, reciprocamente pode aumentar com a maior vulnerabilidade existente nos idosos mais velhos e a possibilidade da presença de múltiplas morbidades.

Existem limitações a serem consideradas para a interpretação e generalização dos dados desta investigação. O instrumento utilizado (IVCF-20) ainda tem uso incipiente, contando com poucos estudos. No entanto, ele é um instrumento de rastreio que permite o reconhecimento precoce dos principais determinantes da saúde dos idosos frágeis e é capaz de proporcionar à equipe da ESF a possibilidade de adotar as medidas protetoras que visam impedir, retardar ou recuperar a autonomia e independência desta população.

É importante considerar ainda que algumas variáveis foram aferidas a partir do relato do próprio idoso ou de familiares, o que pode implicar em vieses decorrentes da limitação da memória humana. Outro ponto é o delineamento transversal desta pesquisa que impossibilita a determinação da casualidade. Finalmente, os idosos entrevistados foram restritos àqueles assistidos por equipes da ESF. Porém, o desenho amostral e o elevado número de idosos envolvidos no estudo garantem representatividade do grupo estudado e legitimam os resultados alcançados, sobretu- do para a população idosa que mais necessita de uma atenção contínua e qualificada.

O município avaliado conta com elevada cobertura de equipes de saúde da Família e dispõe ainda de um Centro de Referência em Atenção à Saúde do Idoso (CRASI). Contudo, não parece existir uma adequada articulação entre os diferentes níveis de atenção da rede ${ }^{45}$. Essa desarticulação da rede na assistência ao idoso corrobora com a necessidade de redesenhar o modelo de atenção ao cuidado ao idoso e do mesmo modo, reforça a necessidade do reconhecimento rápido e antecipado de fragilidade e de seus fatores associados na população idosa atendida pela atenção primária.

Nesta pesquisa detectou-se prevalência significativa de idosos frágeis assistidos pelas equipes da ESF. Os resultados reiteram que o processo do envelhecimento e a multidimensionalidade da fragilidade estão fortemente relacionados e devem continuar como objeto de profundas discussões e reflexões dos pesquisadores e profissionais das diferentes áreas da saúde. A síndrome de fragilidade emerge como um gigante da geriatria e sério problema de saúde pública da sociedade contemporânea. Logo, não podem ser negligenciados alvos de ações preventivas e possíveis alternativas que possibilitam chances para melhor desempenho frente à fragilização do indivíduo.

São necessárias e urgentes ações de planejamento e implementação de cuidados específicos para as demandas dos diferentes estratos clínicofuncionais da fragilidade, bem como das necessidades sociais desta população. $\mathrm{O}$ desenvolvimento de ferramentas de fácil aplicação e de caráter multidimensional é fundamental para envolvimento e ampliação desta discussão para todos os níveis de atenção à saúde. Ao mesmo tempo, torna-se essencial a proposição de programas de cuidados centrados e integrados na pessoa idosa associados à capacitação multiprofissional permanente, que favorece o desenvolvimento de modelo diferenciado de sistema de saúde. 


\section{Colaboradores}

LC Maia participou da concepção, planejamento, análise, interpretação e redação do trabalho. EN Moraes participou da concepção, planejamento, análise, interpretação e redação do trabalho. SM Costa participou da concepção, planejamento, análise, interpretação e redação do trabalho. AP Caldeira participou da concepção, planejamento, análise, na interpretação e redação do trabalho. Ambos os autores aprovaram a versão final encaminhada.

\section{Referências}

1. Veras RP, Oliveira M. Envelhecer no Brasil: a construção de um modelo de cuidado. Cien Saude Colet 2018; 23(6):1929-1936.

2. World Health Organization (WHO). World report on ageing and health. Genebra: WHO; 2015.

3. Ciosak SI, Braz E, Costa MFB, Neves A, Nakano NGR, Rodrigues J, Alencar RA, Rocha ACAL. Senescência e senilidade: novo paradigma na atenção básica de saúde. Rev Esc Enferm USP 2011; 45(2):1763-1768.

4. Fried LP, Tangen CM, Walston J, Newman AB, Hirsch C, Gottdiener J, Seeman T, Tracy R, Kop WJ, Burke G, McBurnie MA. Frailty in older adults: evidence for a phenotype. J Gerontol A Biol Sci Med Sci 2001; 56(3):M146-M156.

5. Rockwood K, Mitnitski A. Frailty defined by deficit accumulation and geriatric medicine defined by frailty. Clin Geriatr Med 2011; 27(1):17-26.

6. Morley JE, Vellas B, Kan GA, Anker SD, Bauer JM, Bernabei R, Cesari M, Chumlea WC, Doehner W, Evans J, Fried LP, Guralnik JM, Katz PR, Malmstrom T, McCartes RJ, Robledo LMG, Rockhood K, Heahling S, Vandewoude MF, Walston J. Frailty consensus: A call to action. J Am Med Dir Assoc 2013; 14(6):392-397.

7. Clegg A, Young J, IIiffe S, Rekkert MO, Rockwood K. Frailty in elderly people. Lancet 2013; 381(9868):752762.

8. Santiago LM, Luz LL, Mattos IE, Gobbens RJ. Cross-cultural adaptation of the Tilburg Frailty Indicator (TFI) for use in the Brazilian population. Cad Saúde Pública 2012; 28(9):1795-1801.

9. Trevisan C, Veronese N, Maggi S, Baggio G, Toffanello ED, Sartori L, Musacchio E, Perissinotto E, Crepaldi G, Manzato E, Sergi G. Factors Influencing Transitions Between Frailty States in Elderly Adults: The Progetto Veneto Anziani Longitudinal Study. J Am Geriat Soc 2017; 65(1):179-184.

10. Chang SF, Lin PL. Frail phenotype and mortality prediction: A systematic review and meta-analysis of prospective cohort studies. Int J Nurs Stud 2015; 52(8):1362-1374.

\section{Agradecimentos}

À Fundação de Amparo à Pesquisa do Estado de Minas Gerais (Fapemig) pelo apoio financeiro e ao Conselho Nacional de Desenvolvimento Científico e Tecnológico (CNPq).
11. Vermeiren S, Vella-Azzopardi R, Beckwée D, Habbig AK, Scafoglieri A, Jansen B, Bautmans I. Frailty and the Prediction of Negative Health Outcomes: A Meta-Analysis J Am Med Dir Assoc 2016; 17(12):1163. e1-17.

12. Barbosa SR, Mansur HN, Colugnati FAB. Impactos da Fragilidade sobre desfechos negativos em saúde de idosos brasileiros. Rev Bras Geriatr Gerontol 2017; 20(6):836-844.

13. Cesari M, Calvani R, Marzetii E. Frailty in Older Persons. Clin Geriatr Med 2017; 33:293-303.

14. Rockwood K, Howlett SE, MacKnight C, Beattie BL, Bergman H, Hebert R, Hogan DB, Wolfson C, McDowell I. Prevalence, attributes, and outcomes of fitness and frailty in community dwelling older adults: report from the Canadian study of health and aging. J Gerontol A Biol Sci Med Sci 2004; 59(12):1310-1317.

15. Brasil. Ministério da Saúde (MS). Diretrizes para o cuidado das pessoas idosas no SUS: proposta de Modelo de Atenção Integral: XXX Congresso Nacional de Secretarias Municipais de Saúde. Brasília: MS; 2014.

16. Santos-Eggimann B, Cuenoud P, Spagnoli J, Junod J. Prevalence of frailty in middle-aged and older community-dwelling Europeans living in 10 countries. J Gerontol A Biol Sci Med Sci 2009; 64(6):675-681.

17. Da Mata FAF, Pereira PPS, Andrade KRC, Figueiredo ACMG, Pereira MG. Prevalence of Frailty in Latin America and the Caribbean: A Systematic Review and Meta-Analysis. PLoS ONE 2016; 11(8):e0160019.

18. Sousa AC, Dias RC, Maciel AC, Guerra RO. Frailty syndrome and associated factors in community dwelling elderly in Northeast Brazil. Arch Gerontol Geriatr 2012; 54:e95-101.

19. Rockwood K, Xiaowei S, Macknight C, Bergman H, Hogan DB, McDowell I, Mitnitski A. A global clinical measure of fitness and frailty in elderly people. CMAJ 2005; 173:489-495.

20. Dent E, Kowal P, Hoogendijk EO. Frailty measurement in research and clinical practice: A review. Eur J Intern Med. 2016; 31:3-10. 
21. Moraes EN, Carmo JA, Moraes FL, Azevedo RS, Machado CJ, Montilla DER. Índice de Vulnerabilidade Clínico Funcional-20 (IVCF-20): reconhecimento rápido do idoso frágil. Rev Saúde Pública 2016; 50:81.

22. Blay SL, Ramos LR, Mari JJ. Validity of a Brazilian version of the Older Americans Resources and Services (OARS) mental health screening questionnaire. J Am Geriat Soc 1988; 36(8):687-692.

23. Vieira RA, Guerra RO, Giacomin KC, Vasconcelos KSS, Andrade ACS, Pereira LSM, Dias JMD, Dias RC. Prevalência de fragilidade e fatores associados em idosos comunitários de Belo Horizonte, Minas Gerais, Brasil: dados do estudo FIBRA. Cad Saúde Pública 2013; 29(8):1631-1643.

24. Carneiro J, Ramos GCF, Barbosa ATF, Mendonça JMG, Costa FM, Caldeira AP. Prevalência e fatores associados à fragilidade em idosos não institucionalizados. Rev Bras Enferm 2016; 69(3):435-442.

25. Fhon JRS, Rodrigues RAP, Santos JLF, Diniz MA, Santos EB, Almeida VC, Giacomini SBL. Factors associated with frailty in older adults: a longitudinal study. Rev Saúde Pública 2018; 52:74-82.

26. Tavares D, Corrêa T, Dias F, Ferreira P, Pegorari M. Frailty syndrome and socioeconomic and health characteristics among older adults. Colomb Med 2017; 48(3):126-131.

27. Canêdo AC, Lopes CS, Lourenço RA. Prevalence of and factors associated with successful aging in Brazilian older adults: Frailty in Brazilian older people Study (FIBRA RJ). Geriatr Gerontol In 2018; 18(8):1280-1285.

28. Closs VE, Ziegelmann PK, Gomes I, Schwanke CHA. Frailty and geriatric syndromes in elderly assisted in primary health care. Acta Sci Health Sci 2016; 38(1):918.

29. Ramos LR, Tavares NUL, Bertoldi AD, Farias MR, Oliveira MA, Luiza VL, Dal Pizzol TS, Arrais PSD, Mengue SS. Polifarmácia e polimorbidade em idosos no Brasil: um desafio para a saúde pública. Rev Saúde Pública 2016; 50(2):9s.

30. Nascimento RCRM, Álvares J, Guerra JAA, Gomes IC, Silveira MR, Costa EA, Leite SN, Costa KS, Soeiro OM, Guibu IA, Karnikowoski MGO, Acurcio FA. Polifarmácia: uma realidade na atenção primária do Sistema Único de Saúde. Rev Saúde Pública 2017; 51(2):19s.

31. Gutiérrez-Valencia $\mathrm{M}$, Izquierdo $\mathrm{M}$, Cesari $\mathrm{M}$, Casas-Herrero Á, Inzitari M, Martínez-Velilla N. The relationship between frailty and polypharmacy in older people: A systematic review. Br J Clin Pharmacol 2018; 84(7):1432-1444.

32. Saum KU, Schöttker B, Meid AD, Holleczek B, Haefeli WE, Hauer K, Brenner H. Is Polypharmacy Associated with Frailty in Older People? Results from the ESTHER Cohort Study. J Am Geriat Soc 2016; 65(2):e27-e32.

33. Diez-Ruiz A, Bueno-Errandonea A, Nuñez-Barrio J, Sanchez-Martín I, Vrotsou K, Vergara E. Factors associated with frailty in primary care: a prospective cohort study. BMC Geriat 2016; 16(91):1-8.

34. Slater N, White S, Venables R, Frisher M. Factors associated with polypharmacy in primary care: a cross-sectional analysis of data from The English Longitudinal Study of Ageing (ELSA). BMJ Open 2018; 8:e020270.
35. Buigues C, Padilha-Sánches C, Garrido JF, Navarro-Martinez R, Ruiz-Ros V, Cauli O. The relationship between depression and frailty syndrome: a systematic review. Aging Ment Health 2015; 9(19):762-772.

36. Tavares DMS, Almeida E, Ferreira PCS, Dias FA, Pegorari MS. Status de fragilidade entre idosos com indicativo de depressão segundo o sexo. J Bras Psiquiatr 2014; 63(4):347-353.

37. Brigola AG, Rossetti ES, Santos BR, Neri AL, Zazzetta MS, Inouye K, Pavarine SCI. Relação entre cognição e fragilidade em idosos: uma revisão sistemática. $D e-$ ment Neuropsychol 2015; 9(2):110-119.

38. Melo DM, Barbosa AJG. O uso do Mini-Exame do Estado Mental em pesquisas com idosos no Brasil: uma revisão sistemática. Cien Saude Colet 2015; 20(12):3865-3876.

39. Faria CA, Lourenço, RA, Ribeiro PCC, Lopes CS. Desempenho cognitivo e fragilidade em idosos clientes de operadora de saúde. Rev Saude Publica 2013; 47(5):923-930.

40. Chamberlaim AM, Rutten LJF, Manemann SM, Bocelo BP, Jacobson DJ, Fan C, Crossardt BR, Roger VL, Sauver JL. Frailty Trajectories in an Elderly Population-Based Cohort. J Am Geriat Soc 2016; 64(2):285292.

41. Carneiro JA, Ramos GCF, Barbosa ATF, Medeiros SM, Lima CA, Costa FM, Caldeira AP. Prevalência e fatores associados à incontinência urinária em idosos não institucionalizados. Cad Saude Colet 2017; 25(3):268277.

42. Ribeiro EG, Matozinhos FP, Guimarães GL, Couto AM, Azevedo RS, Mendoza IYQ. Self-perceived health and clinical-functional vulnerability of the elderly in Belo Horizonte/Minas Gerais. Rev Bras Enferm 2018; 71(2):860-867.

43. Medeiros SM, Silva LSR, Carneiro JA, Ramos GCF, Barbosa ATF, Caldeira AP. Fatores associados à autopercepção negativa da saúde entre idosos não institucionalizados de Montes Claros, Brasil. Cien Saude Colet 2016; 21(11):3377-3386.

44. Collard RM, Boter H, RA Schoevers, Oude Voshaar RC. Prevalência da fragilidade em idosos residentes na comunidade: uma revisão sistemática. J Am Geriatr Soc 2012; 60(8):1487-1492.

45. Costa SM, Ferreira A, Xavier LR, Guerra PNS, Rodrigues ACQ. Referência e contrarreferência na saúde da família: percepção dos profissionais de saúde. Revista APS 2013; 16(3):287-293.

Artigo apresentado em 28/11/2018

Aprovado em 18/04/2019

Versão final apresentada em 20/04/2019 